\title{
A Cyanide-bridged Fe(III)-Mn(III) Chain with Metamagnetic Properties and Significant Magnetic Anisotropy
}

\author{
Shi Wang, ${ }^{*}$ Marilena Ferbinteanu, ${ }^{*, 1}$ and Masahiro Yamashita \\ Department of Chemistry, Graduate School of Science, Tohoku University, \\ Aramaki, Aoba-ku, Sendai 980-8578, Japan and \\ CREST, Japan Science and Technology Agency (JST), Japan \\ E-mail: swang@agnus.chem.tohoku.ac.jp; marilena@agnus.chem.tohoku.ac.jp
}

\section{Supporting materials}

\section{Experimental \\ II. Molecular and Crystal Structure \\ III. Spin Hamiltonian Models and Magnetic Susceptibility Fit IV. Broken Symmetry DFT Calculation Details}

\footnotetext{
${ }^{1}$ Permanent address: University of Bucharest, Inorg. Chem. Lab, Dumbrava Rosie 23, Bucharest 70254, Romania, mcimpoesu@yahoo.com
} 


\section{Experimental:}

A solution of $\left(\mathrm{Bu}_{4} \mathrm{~N}\right)\left[(\mathrm{Tp}) \mathrm{Fe}(\mathrm{CN})_{3}\right]\left(\mathrm{Bu}_{4} \mathrm{~N}^{+}=\right.$tetrabutylammonium cation) $(71 \mathrm{mg}, 0.12$ mmol) in $10 \mathrm{ml}$ of methanol was added to a solution of $\left[\mathrm{Mn}(5-\mathrm{MeOsalen})\left(\mathrm{H}_{2} \mathrm{O}\right)\right]\left(\mathrm{PF}_{6}\right)^{9}$ (63 mg, $0.12 \mathrm{mmol}$ ) in $10 \mathrm{ml}$ of methanol, and the mixture was stirred for $5 \mathrm{~min}$ and left undisturbed. After a week, dark brown crystals of 1 were obtained in a yield of $62 \%$.

Characterization of 1: IR (solid, $\mathrm{KBr}$ pellet): $v_{\mathrm{CN}} 2115$ (w), 2129 (m). Anal. Calcd for $\mathrm{C}_{32} \mathrm{H}_{36} \mathrm{BN}_{11} \mathrm{O}_{6} \mathrm{MnFe}: \mathrm{C}, 48.51 ; \mathrm{H}, 4.58 ; \mathrm{N}, 19.45$. Found: C, 47.75; H, 4.44; N, 20.05.

The $\mathrm{C} \equiv \mathrm{N}$ stretching in the infrared spectrum of $\mathbf{1}$ is consistent with the presence of bridging (a medium peak at $2129 \mathrm{~cm}^{-1}$ ) and terminal (a weak peak at $2115 \mathrm{~cm}^{-1}$ ) cyanides. 


\section{Molecular and Crystal Structure}
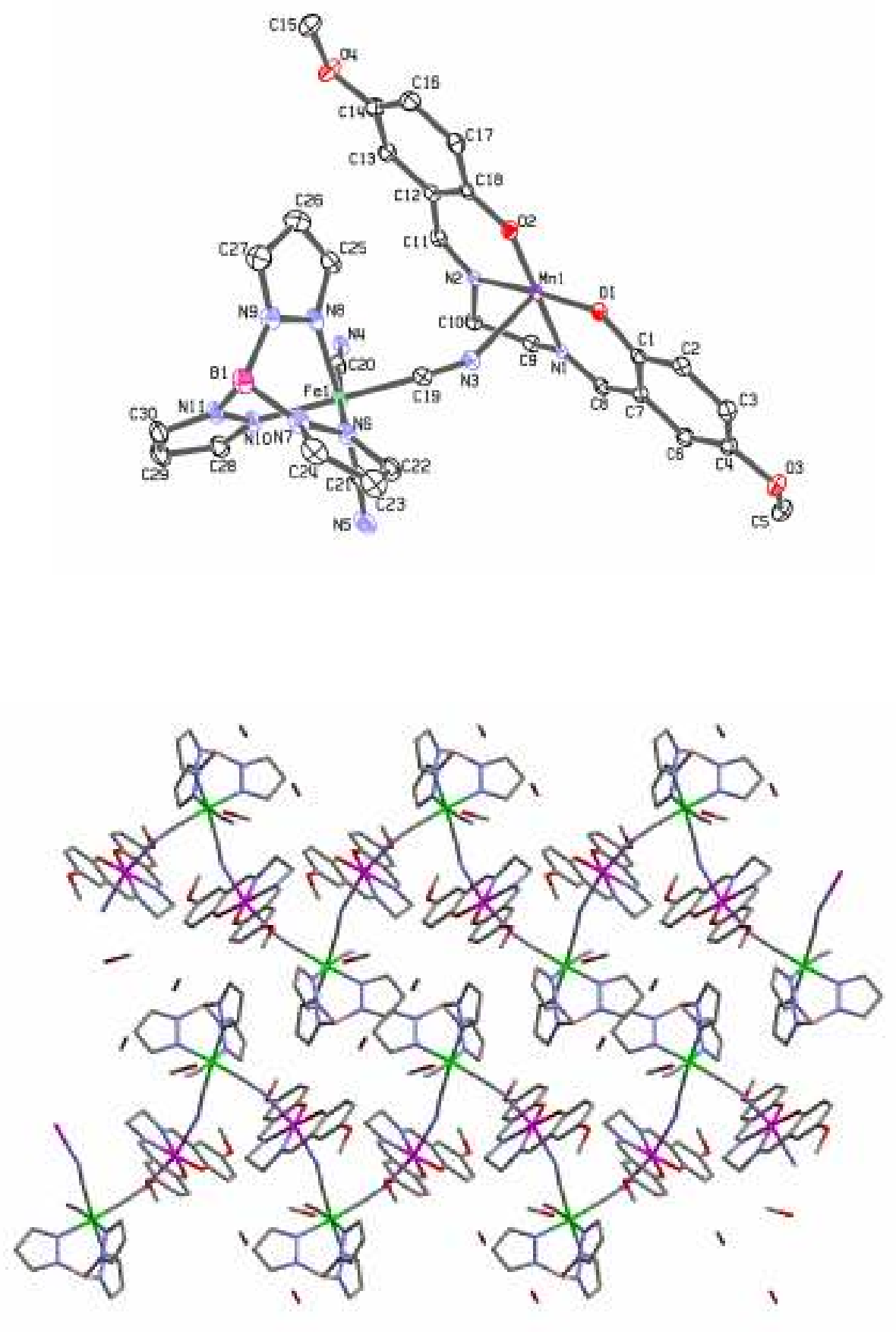

(a) 

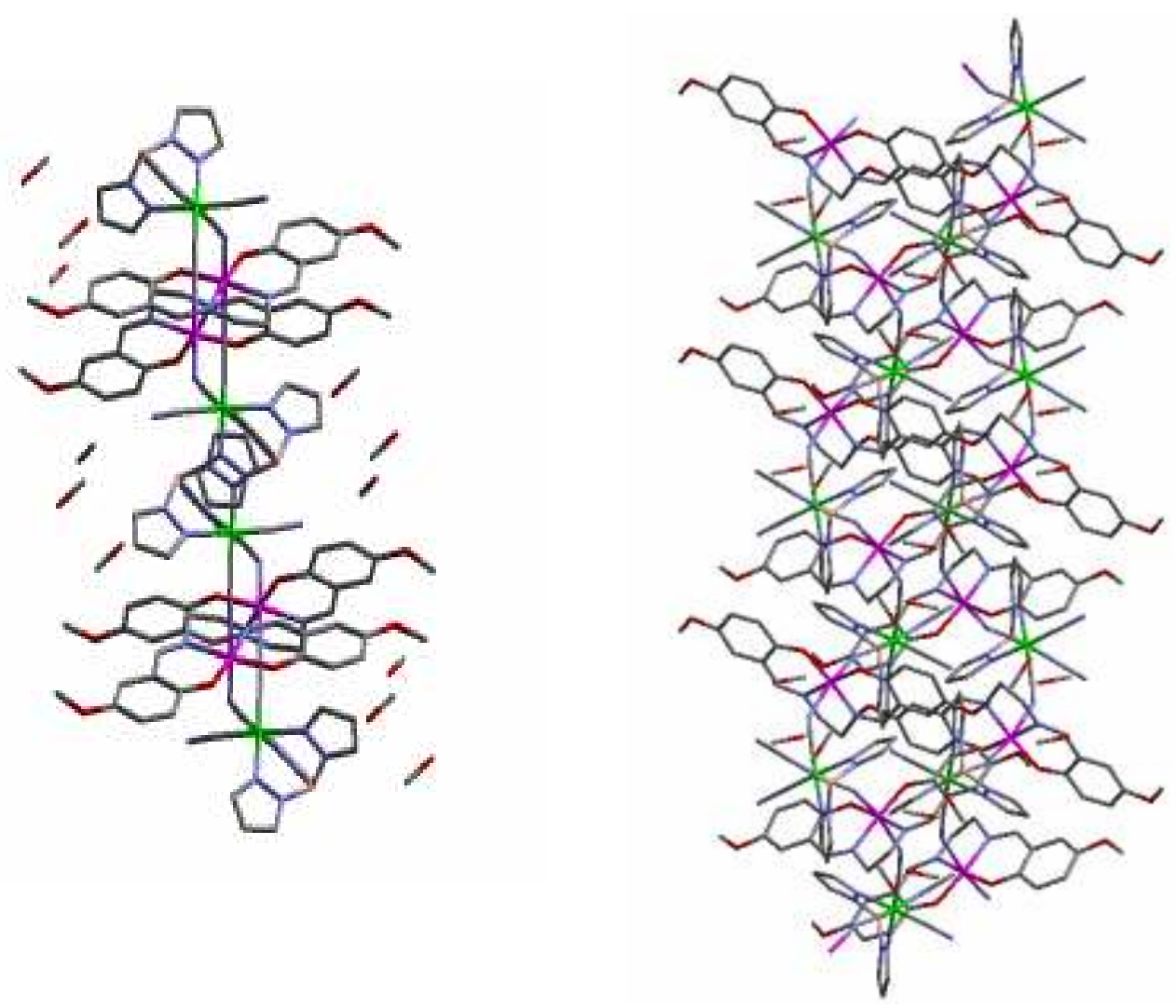

(b)

(c)

Figure S1. Molecular structure of $\mathbf{1}$ (top), the solvated methanol molecules and hydrogen atoms have been omitted for clarity (ORTEP, 50\% ellipsoids). The zigzag chains view along $a, b, c$ directions (bottom). Selected bond lengths and angles: $\mathrm{Fe}(1)-\mathrm{C}(19)=$ 1.920(3) $\AA, \mathrm{Fe}(1)-\mathrm{C}(20)=1.927(3) \AA, \mathrm{Fe}(1)-\mathrm{C}(21)=1.933(3) \AA, \mathrm{Mn}(1)-\mathrm{N}(1)=1.986(2)$ $\AA, \mathrm{Mn}(1)-\mathrm{N}(2)=1.980(2) \AA, \mathrm{Mn}(1)-\mathrm{O}(1)=1.873(2) \AA, \mathrm{Mn}(1)-\mathrm{O}(2)=1.863(2) \AA$, $\mathrm{Mn}(1)-\mathrm{N}(3)=2.320(2) \AA, \mathrm{Mn}(1)-\mathrm{N}(4) \# 1=2.364(2) \AA, \mathrm{Fe}(1)-\mathrm{C}(21) \equiv \mathrm{N}(5)=174.5(3)^{\circ}$, $\mathrm{Fe}(1)-\mathrm{C}(19) \equiv \mathrm{N}(3)=174.4(3)^{\circ}, \mathrm{Fe}(1)-\mathrm{C}(20) \equiv \mathrm{N}(4)=175.7(3)^{\circ}, \mathrm{Mn}(1)-\mathrm{N}(3) \equiv \mathrm{C}(19)=$ 151.1(2) ${ }^{\circ}, \mathrm{Mn}(1)-\mathrm{N}(4) \# 1 \equiv \mathrm{C}(20) \# 1=161.7(2)^{\circ}, \mathrm{N}(3)-\mathrm{Mn}(1)-\mathrm{N}(4) \# 1=167.47(9)^{\circ}(\# 1$ : $1.5-\mathrm{x}, 1 / 2+\mathrm{y}, 1 / 2-\mathrm{z})$. 


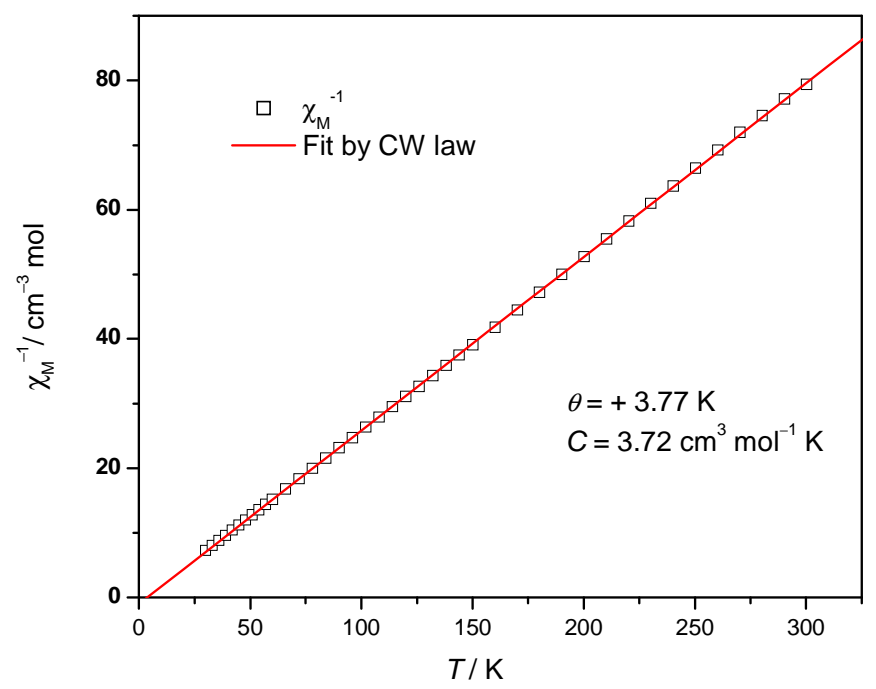

Figure S2. Temperature dependence of $\chi_{\mathrm{M}}{ }^{-1}$ for $\mathbf{1}$.

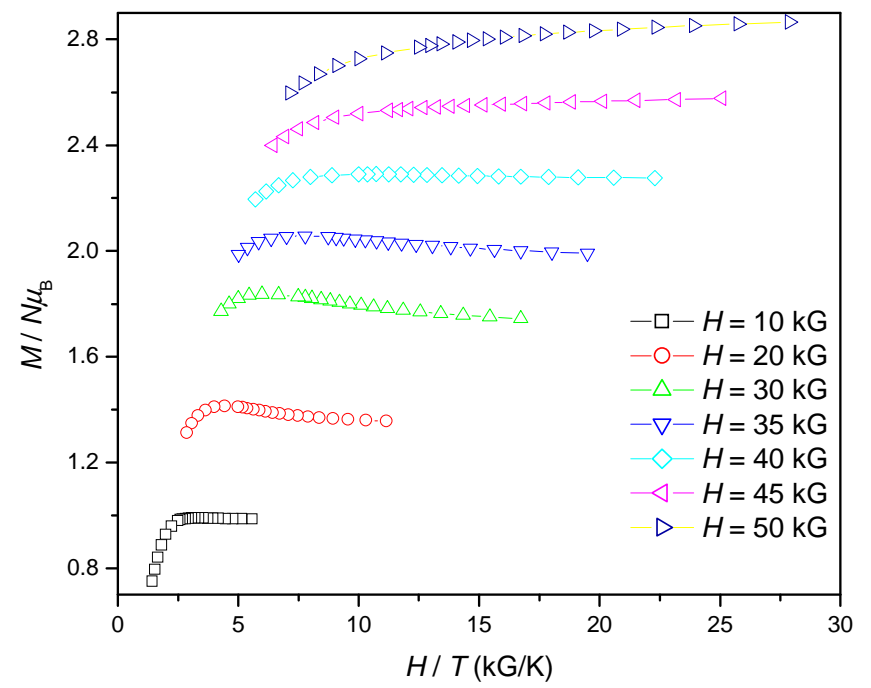

Figure S3. Reduced magnetization, $M / N_{A} \mu_{\mathrm{B}}\left(N_{A}\right.$ is Avogadro's number and $\mu_{\mathrm{B}}$ is Bohr magneton) versus $H / T$ for 1 . Data were measured in the $1.8-7.0 \mathrm{~K}$ range and at seven magnetic fields. 


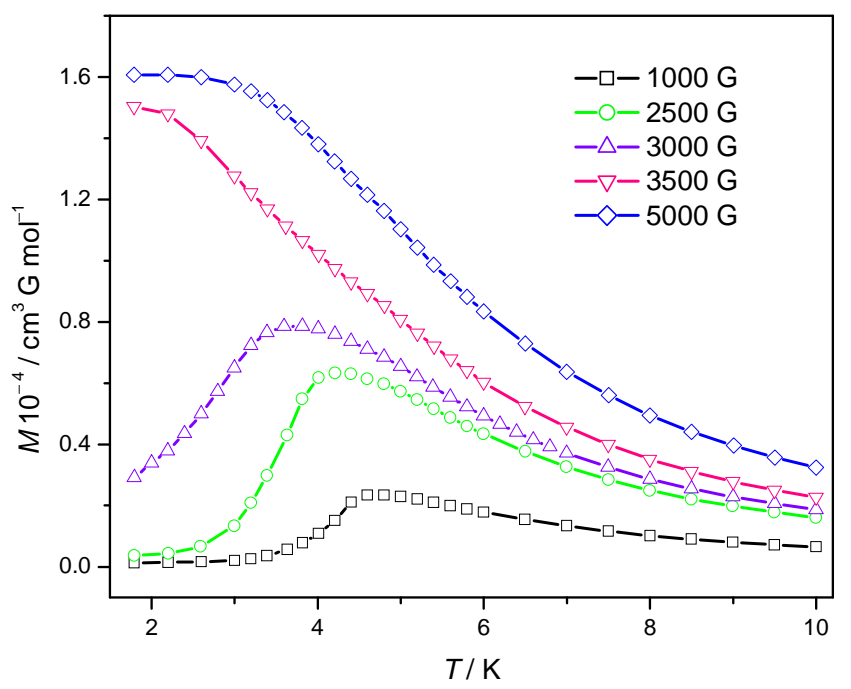

Figure S4. Field-cooled magnetization for $\mathbf{1}$ at different applied fields.

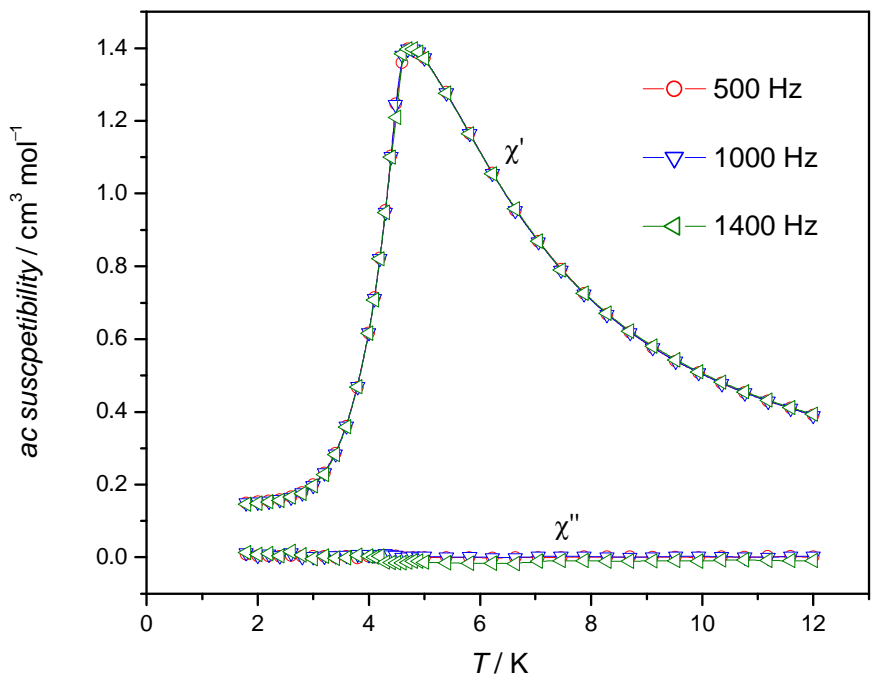




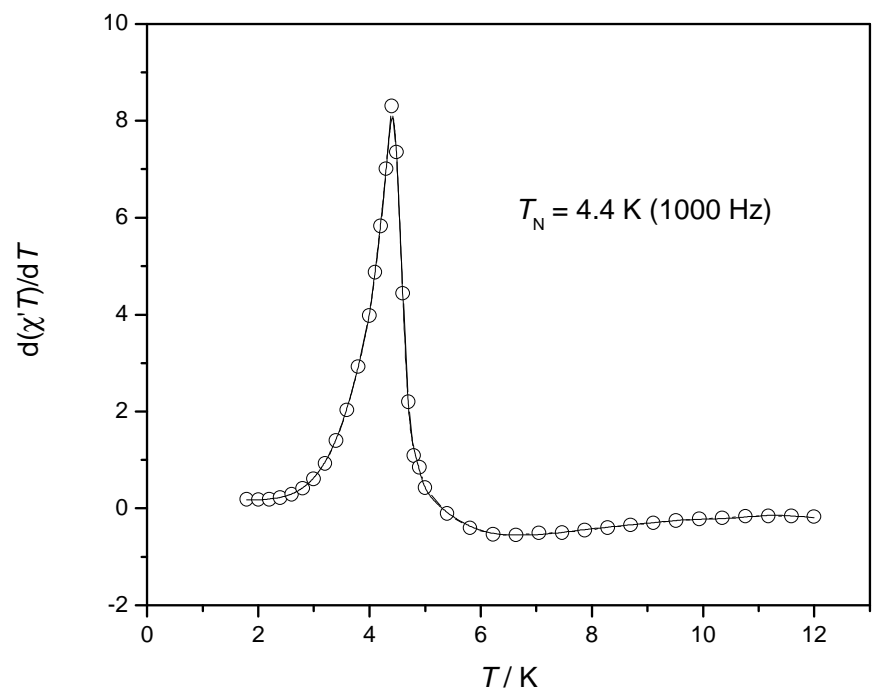

Figure S5. In-phase $\left(\chi^{\prime}\right)$ and out-of-phase $\left(\chi^{\prime \prime}\right)$ ac susceptibility in zero applied dc field and an ac field of $3 \mathrm{G}$ at different frequencies (top) and $\mathrm{d}\left(\chi^{\prime} T\right) / \mathrm{d} T$ at $1000 \mathrm{~Hz}$ (bottom) for 1. 


\section{Spin Hamiltonian Models and Magnetic Susceptibility Fit}

The general zero-field splitting (ZFS) and Heisenberg Dirac van Vleck (HDVV) Hamiltonian of the anisotropic alternating chain can be ascribed as:

$$
\hat{H}=\sum_{i=1}^{\infty} D\left(\left(\hat{S}_{2 i-1}^{z}\right)^{2}-2\right)+\sum_{i=1}^{\infty}\left(-2 J_{a} \hat{S}_{2 i} \cdot \hat{S}_{2 i+1}-2 J_{b} \hat{S}_{2 i-1} \cdot \hat{S}_{2 i}-2 J^{\prime} \hat{S}_{2 i-1} \cdot \hat{S}_{2 i+1}\right)
$$

where the $\mathrm{Mn}(\mathrm{III})$ centers take the odd labels $(2 i-1,2 i+1 \ldots)$ and the $\mathrm{Fe}(\mathrm{III})$ ones the even indices (2i). The alternating chain consists in $J_{a}$ and $J_{b}$ parameters, as described in the main text and depicted in the next schemes. A $J^{\prime}$ parameter can be assigned for the nextneighbor interaction between the $\mathrm{Mn}(\mathrm{III})$, on the ground of the fact that their 5-MeOsalen ligands fall in rather close contacts due to the zigzag geometry. Taking precautionary inquiries, the $J^{\prime}$ parameter was a priori considered in our approach, but a posteriori it was concluded that, tuned in a small magnitude of both ferro and antiferro range, it cannot change the overall $\chi T$ shape significantly. Particularly, the $J^{\prime}$ has, conceivably, the same magnitude range as the mean field $z J$ intermolecular parameter, but its variation was ultimately proved of lesser impact. The $J^{\prime}$ was discarded in the final model and results report, but, for sake of completeness, it was kept in the general schemes described here, as well in the computation tests from next section in this text.

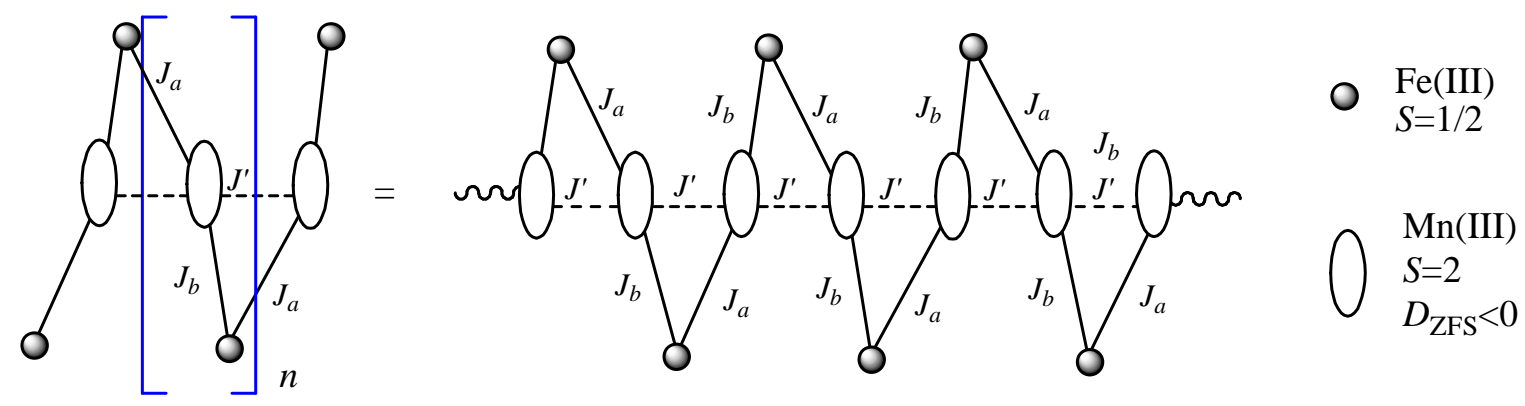

\section{Scheme S1.}

To make the fit tractable, smaller sequences must be taken. The $n=3$ limitation is an appropriate one. There are two types of open chain fragment cuts: A: a structure containing $2 \times J_{a}, 3 J_{b}, 2 \times J^{\prime}, \mathrm{B}$ : a structure containing $3 \times J_{a}, 2 \times J_{b}, 2 \times J^{\prime}$, and a cyclic structure containing C: $3 \mathrm{x}_{a}, 3 \mathrm{x}_{b}, 3 \mathrm{xJ}^{\prime}$ couplings. 


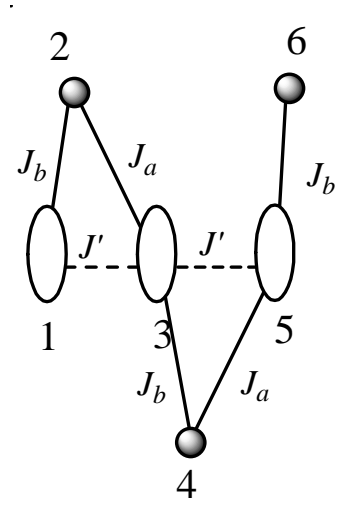

A

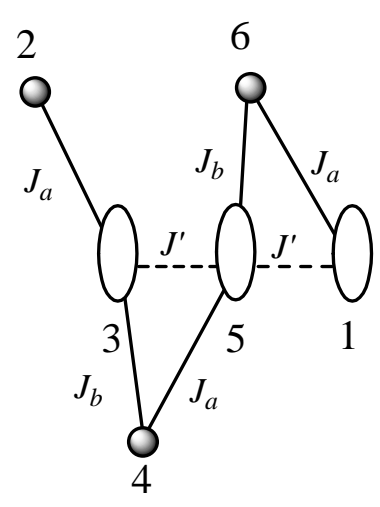

B

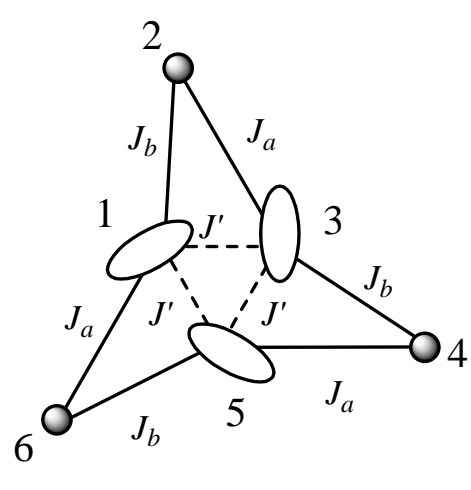

$\mathrm{C}$

\section{Scheme S2.}

The cyclic structure was taken to enforce the convergence toward the periodicity condition. The corresponding fragment Spin Hamiltonians associated to the above schemes are as follows:

$$
\begin{aligned}
\hat{H}_{A} & =D\left(\left(\hat{S}_{1}^{z}\right)^{2}+\left(\hat{S}_{3}^{z}\right)^{2}+\left(\hat{S}_{5}^{z}\right)^{2}-6\right) \\
& -2 J_{a}\left(\hat{S}_{2} \cdot \hat{S}_{3}+\hat{S}_{4} \cdot \hat{S}_{5}\right)-2 J_{b}\left(\hat{S}_{1} \cdot \hat{S}_{2}+\hat{S}_{3} \cdot \hat{S}_{4}+\hat{S}_{5} \cdot \hat{S}_{6}\right)-2 J^{\prime}\left(\hat{S}_{1} \cdot \hat{S}_{3}+\hat{S}_{3} \cdot \hat{S}_{5}\right) \\
\hat{H}_{B}=D\left(\left(\hat{S}_{1}^{z}\right)^{2}+\left(\hat{S}_{3}^{z}\right)^{2}+\left(\hat{S}_{5}^{z}\right)^{2}-6\right) & \\
& -2 J_{a}\left(\hat{S}_{2} \cdot \hat{S}_{3}+\hat{S}_{4} \cdot \hat{S}_{5}+\hat{S}_{6} \cdot \hat{S}_{1}\right)-2 J_{b}\left(\hat{S}_{5} \cdot \hat{S}_{6}+\hat{S}_{3} \cdot \hat{S}_{4}\right)-2 J^{\prime}\left(\hat{S}_{3} \cdot \hat{S}_{5}+\hat{S}_{5} \cdot \hat{S}_{1}\right) \\
\hat{H}_{C} & =D\left(\left(\hat{S}_{1}^{z}\right)^{2}+\left(\hat{S}_{3}^{z}\right)^{2}+\left(\hat{S}_{5}^{z}\right)^{2}-6\right) \\
& -2 J_{a}\left(\hat{S}_{2} \cdot \hat{S}_{3}+\hat{S}_{4} \cdot \hat{S}_{5}+\hat{S}_{6} \cdot \hat{S}_{1}\right)-2 J_{b}\left(\hat{S}_{1} \cdot \hat{S}_{2}+\hat{S}_{3} \cdot \hat{S}_{4}+\hat{S}_{5} \cdot \hat{S}_{6}\right)-2 J^{\prime}\left(\hat{S}_{1} \cdot \hat{S}_{3}+\hat{S}_{3} \cdot \hat{S}_{5}+\hat{S}_{5} \cdot \hat{S}_{1}\right)
\end{aligned}
$$

The magnetic susceptibility subjected to the fit was taken as the average of those computed on the above fragments.

$$
(\chi T)_{\text {calc }}=\frac{1}{3}\left((\chi T)_{A}+(\chi T)_{B}+(\chi T)_{C}\right)
$$

The fit was initiated with 50000 random sets $\left\{D, J_{\mathrm{a}}, J_{b}, J^{\prime}, z J\right\}$ placed respectively in the interval $\{-1: 6,0: 6,0: 6,-0.3: 0.3,-0.3: 0.1\} \mathrm{cm}^{-1}$, selecting the best fitting random choice as start for the iterative fit. 
The regular mean field implying the $z J$ parameter can be applied according to the following formulas:

$$
\begin{aligned}
& \chi_{M F}=\frac{\chi_{\text {calc }}}{1-\frac{z J}{N_{A} \mu_{B}^{2} g^{2}} \chi_{\text {calc }}} \\
& \chi_{M F} T=\frac{\chi_{\text {calc }} T}{1-\frac{1}{T} \cdot \frac{z J}{N_{A} \mu_{B}^{2} g^{2}}\left(\chi_{\text {calc }} T\right)}
\end{aligned}
$$

where $N_{A}$ is Avogadro's number and $\mu_{\mathrm{B}}$ is Bohr magneton.

The above expressions are equivalent transcriptions of the formula 6.5 .9 in the reference Kahn, O. Molecular Magnetism, Wiley-VCH, NewYork, 1993, p.131. However, as shown in the text, the regular mean field cannot account well for the actual $\chi T$ shape at low $T$, namely a sharp and high peak, being able to produce only rather shallow maxima. In fact, the mean field approach is a rather crude surrogate for the general account of intermolecular magnetic coupling.

E.g., for the $z J>0$ case, the mean field correction produces an abrupt raising of $\chi T$ when $T$ is decreased, down to a divergence point, $z J \cdot \chi_{\text {calc }} / N_{A} \mu_{B}^{2} g^{2} \rightarrow 1$. Thus, assuming that at a given low temperature, the molecular subsystem reached already the groundstate ferromagnetic limit of the spin $S_{\mathrm{g}}$, with $\left(\chi_{\text {calc }} T\right)_{T \rightarrow 0} \rightarrow N \mu_{B}^{2} g^{2} S_{g}\left(S_{g}+1\right) / 3 k_{B}$, one may see that the divergence occurs when $3 k_{B} T_{x} \rightarrow z J \cdot S_{g}\left(S_{g}+1\right)$. Below the $T_{x}$ point, when the denominator becomes negative, the $z J>0$ mean field case leads to drastically meaningless situation, since the $\chi T$ appears negative. Thus, the $+\infty$ divergence on the right side of the $T_{x}$ critical point is useful to mimic the ferromagnetic lattice ordering, but on the left side, the divergence to $-\infty$ and the $\chi T<0$ prove the intrinsic limitations of the mean field concept.

For the $z J<0$ case, the limitations of the mean field are less evident, but still important. Thus, there is a rather smooth convergence to $\chi T \rightarrow 0$ with $T \rightarrow 0$, apparently in line with the requirements of the supramolecular full antiferromagnetic ordering. The $\chi T$ vanishing at low $T$ comes from the $1+$ const $/ T \rightarrow \infty$ limit of the denominator in $\left(6^{\prime}\right)$. This dependence is a progressive attenuation of the $\chi_{\text {calc }} T$ function, having a shallow profile, not a sharp one. Thus, it cannot account for the desiderata of reproducing magnetic transition sharp profiles.

The transitions conceivable as level crossing effect need a treatment that consider at least two interacting units (chains), the regular mean field model remaining however confined to the one-molecular unit description. The text described Ising-like equating of interactions limited between mutually degenerate states among two chains is an intermediate point between crude mean field and full interchain Ising formulas. The Ising terms stands here for the use of spin projections in designing Hamiltonian. Note that the conceived states are however collective ones on the molecular unit (i.e. chain, here) and not the transition metal localized spin projection. However, qualitatively, one may say that the resonant interaction of levels is due to genuine Ising terms between relatively close anisotropic magnetic centers from neighbor chains. The unique $z J$ coupling constant is a phenomenological simplification. Even assigning splitting on all the $i$ chain states, the key factor is practically the groundstate splitting. The splitting effects are already 
smeared out to the interchain paramagnetic limit (equivalent to one non interacting chain only) when $T$ raises sufficiently. The design of the intermolecular interaction and the groundstate level crossing effects, which explain in the actual model the metamagnetic type behavior, are sketched in the next schemes (S3 and S4).

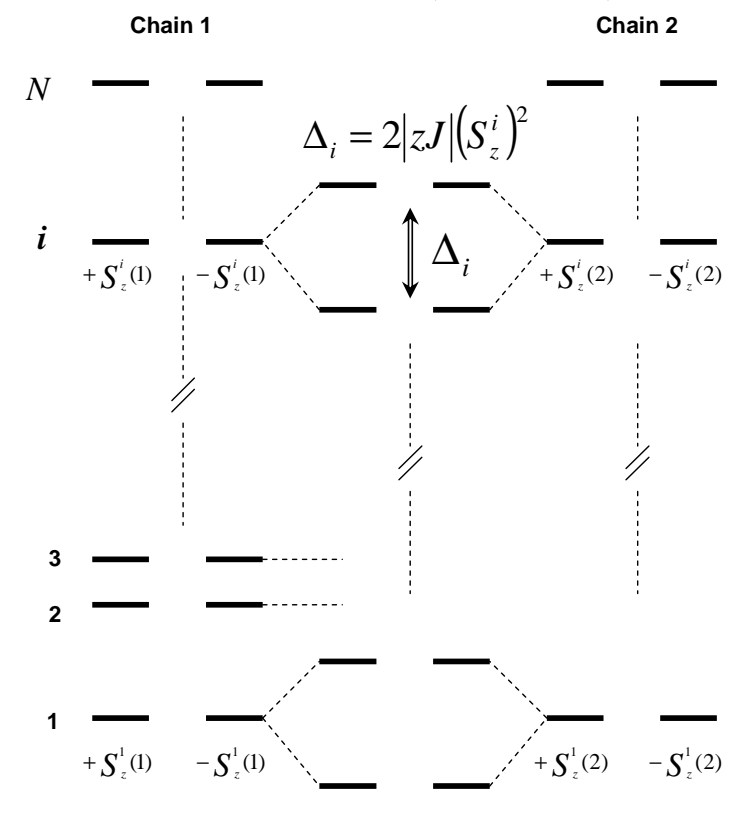

Scheme S3.
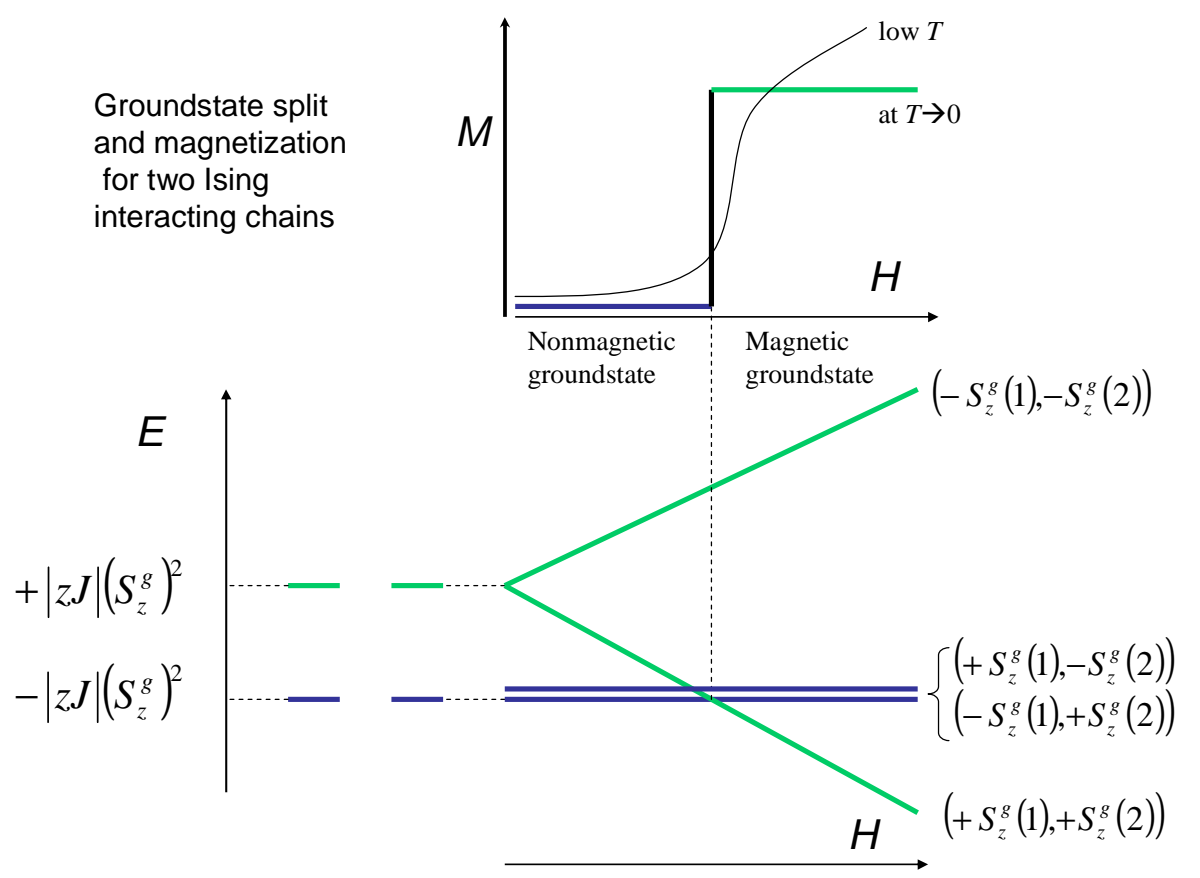

Scheme S4. 
Technically, the procedure is as follows. Each $A, B$ or $C$ above considered spin topology provides series of eigenvalues resulted from their $S_{z}$ block Hamiltonians, labeled formally $E_{i}^{P, S_{z}}$ (with $P=A, B, C$ ). Due to the anisotropy induced by the $D$ axial ZFS term, such values are obtained in degenerate pairs, the blocks with opposite projection, $\pm S_{z}$, having the same series of eigenvalues. The phenomenological model conceives a system made of two chains, as minimal entity to mimic antiferomagnetic ordering in the lattice. Each molecular unit $P$, enters with $N$ total spin states, and the collective $2 N$ states can be ascribed in the following order, keeping the related energy levels in series of 4 successive elements. The four states, $E_{i}^{P,+S_{z}}(1), E_{i}^{P,-S_{z}}(1), E_{i}^{P,+S_{z}}(2), E_{i}^{P,-S_{z}}$ (2) (with (1) and (2) standing for the two $P$ units labels) are degenerate, with $E_{i}^{P|| S_{z} \mid}$ identical values, interacting in resonant regime, via the $-z J \cdot S_{z}^{i}(1) S_{z}^{i}(2)$ Hamiltonian term. Thus, this model is an intermediate step, between a mean field approach, i.e. $-z J \cdot S_{z}^{i}(1)<S_{z}^{i}>$, where $\left\langle S_{z}^{i}\right\rangle$ can be interpreted in context as the average over the $S_{z}^{i}(2)$ spin projections (or generally, over the all neighbors projections) and a full Ising expansion $-z J_{i j} \cdot S_{z}^{i}(1) S_{z}^{j}(2)$ (with both $i$ and $j$ running on $N$ states, producing $N^{2}$ spin states, which is prohibitive and finally useless to build a model dedicated to weak band structure effects). The fact that we consider a single $z J$ parameter is also a technical phenomenological simplification, instead of a $z J_{i}$ for each state, in $-z J_{i} \cdot S_{z}^{i}(1) S_{z}^{i}(2)$ terms. Even considering the splitting on each level of molecular $P$ eigenvalues, the effectively important are the lowest ones (in extreme, only those of the groundstate).

Under appropriate labeling of the states originating from former degenerate isolate two units, the collective energies of the system made of the $(1,2)$ couple can be ascribed as follow:

$$
\begin{aligned}
& E_{4 i-3}^{P}(1,2)=E_{i}^{P,\left|S_{z}\right|}-z J \cdot\left(+S_{z}(1)\right) \cdot\left(+S_{z}(2)\right)=E_{i}^{P,\left|S_{z}\right|}-z J \cdot S_{z}^{2} \\
& E_{4 i-2}^{P}(1,2)=E_{i}^{P,\left|S_{z}\right|}-z J \cdot\left(-S_{z}(1)\right) \cdot\left(-S_{z}(2)\right)=E_{i}^{P,\left|S_{z}\right|}-z J \cdot S_{z}^{2} \\
& E_{4 i-1}^{P}(1,2)=E_{i}^{P,\left|z_{z}\right|}-z J \cdot\left(+S_{z}(1)\right) \cdot\left(-S_{z}(2)\right)=E_{i}^{P,\left|S_{z}\right|}+z J \cdot S_{z}^{2} \\
& E_{4 i}^{P}(1,2)=E_{i}^{P,\left|z_{z}\right|}-z J \cdot\left(-S_{z}(1)\right) \cdot\left(+S_{z}(2)\right)=E_{i}^{P,\left|S_{z}\right|}+z J \cdot S_{z}^{2}
\end{aligned}
$$

The states have summed spin projections:

$$
\begin{aligned}
& \left(S_{z}\right)_{4 i-3}^{P}(1,2)=+S_{z}(1)+S_{z}(2)=+2 S_{z} \\
& \left(S_{z}\right)_{4 i-2}^{P}(1,2)=-S_{z}(1)-S_{z}(2)=-2 S_{z} \\
& \left(S_{z}\right)_{4 i-1}^{P}(1,2)=+S_{z}(1)-S_{z}(2)=0 \\
& \left(S_{z}\right)_{4 i}^{P}(1,2)=-S_{z}(1)+S_{z}(2)=0
\end{aligned}
$$

In the above conventional labeling the $i$ runs from 1 to $N / 2$, so that the maximal $4 i$ index reaches the $2 N$ total of states. With respect of molecular units, the reduction to $N / 2$ comes from the fact that the $N$ states can be ordered in two $N / 2$ mutually degenerate series from the $\pm S_{z}$ companion blocks. 
Working with maximal and minimal spin projection only, discarding the intermediate ones, is somewhat similar to the Broken Symmetry concept in molecular electron structure procedures. The meaning corresponds to the fact that the minimal projection includes an average over all the intermediate spin states resulting from the full sets of mutual products between local wavefunctions. This phenomenological frame is sufficient and adequate for the aimed description.

With energies and spin projections of the designed states one may take the magnetization and susceptibility of the $(1,2)$ molecular couple of $P$ units, as follows, multiplying implicitly with a $1 / 2$ factor to scale back at the conventional value for one $P$ unit embedded in the interacting frame.

$$
\begin{aligned}
M_{P}^{\text {int }}(H, T) & =\frac{1}{2} \cdot g \mu_{B} \cdot \frac{\sum_{r=1}^{2 N} S_{z}^{r}(1,2) \operatorname{Exp}\left(-\frac{E_{z}^{r}(1,2)+g \mu_{B} S_{z}^{r}(1,2) \cdot H}{k_{B} T}\right)}{\sum_{r=1}^{2 N} \operatorname{Exp}\left(-\frac{E_{z}^{r}(1,2)+g \mu_{B} S_{z}^{r}(1,2) \cdot H}{k_{B} T}\right)} \\
(\chi T)_{P}^{\text {int }} & =\frac{1}{2} \cdot \frac{N_{A} g^{2} \mu_{B}^{2}}{k_{B} T} \cdot \frac{\sum_{r=1}^{2 N}\left(S_{z}^{r}(1,2)\right)^{2} \operatorname{Exp}\left(-\frac{E_{z}^{r}(1,2)}{k_{B} T}\right)}{\sum_{r=1}^{2 N} \operatorname{Exp}\left(-\frac{E_{z}^{r}(1,2)}{k_{B} T}\right)}
\end{aligned}
$$

After performing the above handling for the three $P=A, B$, and $C$ defined units, one takes the average, to obtain the trend toward the infinite chain simulation.

$$
\begin{gathered}
(\chi T)_{\text {calc }}^{\mathrm{int}}=\frac{1}{3}\left((\chi T)_{A}^{\mathrm{int}}+(\chi T)_{B}^{\mathrm{int}}+(\chi T)_{C}^{\mathrm{int}}\right) \\
M_{\text {calc }}^{\mathrm{int}}=\frac{1}{3}\left(M_{A}^{\mathrm{int}}+M_{B}^{\mathrm{int}}+M_{C}^{\mathrm{int}}\right)
\end{gathered}
$$




\section{Broken Symmetry DFT Calculation Details}

The calculations were performed on adequate oligomer sequences taken at experimental geometry. The $[\mathrm{Li}(\mu-\mathrm{NC}) \mathrm{FeTp}(\mathrm{CN})(\mu-\mathrm{CN}) \mathrm{Mn}(5-\mathrm{MeOsalen})(\mu-\mathrm{NC}) \mathrm{Li}]^{2+}$ dimers were taken in the two distinct forms corresponding to the $a$ or $b$ sequences as described in the text. The $\mathrm{Li}^{+}$ions were added at the terminal $\mathrm{CN}$ groups to mimic in the discrete structure, the polarization effects of the next metal ion members of the complete chain. The $\left[\mathrm{CoTp}(\mathrm{CN})_{3}(\mathrm{Mn}(5-\mathrm{MeOsalen}) \mathrm{NCLi})_{2}\right]^{+}$trimer, with the $\mathrm{Co}$ (III) diamagnetic center replacing the Fe(III) was used to estimate the distant $\mathrm{Mn}(\mathrm{III})-\mathrm{Mn}$ (III) interaction, through 5-MeOSalen van der Waals contacts.

The 6-311G* basis set was taken for the metal ions and the coordinating atoms $(\mathrm{N}, \mathrm{O}$ and $\mathrm{C}$ ). The $\mathrm{C}$ and $\mathrm{H}$ atoms pertaining to the ligand skeleton were taken with the 6-31G basis. The unrestricted B3LYP DFT calculations were performed with the Gaussian 03 code. $^{2}$

The Broken Symmetry (BS) treatment implied careful series of calculations that, by subsequent localization and orbital reordering procedures prepared the appropriate starting point of the BS type. The corresponding spin flip in the HS vs. LS unrestricted DFT single determinants is not obtained automatically, without the preliminary ordering care. A test of the BS handling is the closeness of the computed Mulliken spin populations to the spin-flip relationship when compare the Broken High Spin (BHS) and Broken Low Spin (BLS) configurations output. We pedantically recall that in the BS approach, i.e. the single determinant frame the BLS configuration does not correspond to the lowest $S$, but formally to an average of all spin energy levels, while BHS is somewhat parallel to the maximal $S$ state, though in the unrestricted calculation the $S$ cannot be called rigorous quantum number. Besides the reported general performance, the BS technique is a tool that can be employed, complementary to the experiment, in a rather user friendly manner, with the pursuit of chemical intuition. The Yamaguchi and Onishi formula ${ }^{3}$ was used to handle the Broken Symmetry DFT output.

$$
J=-\left(E_{B H S}-E_{B L S}\right) /\left[\left\langle\hat{S}^{2}\right\rangle_{B H S}-\left\langle\hat{S}^{2}\right\rangle_{B L S}\right]
$$

(2)Gaussian 03, Frisch, M. J.; Trucks, G. W. ; Schlegel, H. B.; Scuseria, G. E. ; Robb, M. A.; Cheeseman, J. R.; Montgomery, Jr., J. A.; Vreven, T.; Kudin, K. N. ; Burant, J. C. ; Millam, J. M.; Iyengar, S. S. ; Tomasi, J. ; Barone, V. ; Mennucci, B. ; Cossi, M.; Scalmani, G. ; Rega, N. ; Petersson, G. A.; Nakatsuji, H. ; Hada, M.; Ehara, M.; Toyota, K. ; Fukuda, R.; Hasegawa, J. ; Ishida, M.; Nakajima, T. ; Honda, Y. ; Kitao, O.; Nakai, H. ; Klene, M.; Li, X.; Knox, J. E. ; Hratchian, H. P. ; Cross, J. B. ; Adamo, C.; Jaramillo, J. ; Gomperts, R. ; Stratmann, R. E.; Yazyev, O. ; Austin, A. J. ; Cammi, R. ; Pomelli, C.; Ochterski, J. W. ; Ayala, P. Y. ; Morokuma, K.; Voth, G. A.; Salvador, P.; Dannenberg, J. J. ; Zakrzewski, V. G. ; Dapprich, S. ; Daniels, A. D. ; Strain, M. C.; Farkas, O. ; Malick, D. K. ; Rabuck, A. D. ; Raghavachari, K.; Foresman, J. B. ; Ortiz, J. V.; Cui, Q.; Baboul, A. G. ; Clifford, S. ; Cioslowski, J. ; Stefanov, B. B.; Liu, G. ; Liashenko, A. ; Piskorz, P. ; Komaromi, I. ; Martin, R. L.; Fox, D. J. ; Keith, T. ; Al-Laham, M. A. ; Peng, C. Y. ; Nanayakkara, A. ; Challacombe, M.; Gill, P. M. W. ; Johnson, B. ; Chen, W. ; Wong, M. W. ; Gonzalez, C. and Pople, J. A. ; Gaussian, Inc., Pittsburgh PA, 2003.

(3) (a) Mitani, M. ; Mori, H. ; Takano, Y.; Yamaki, D.; Yoshioka, Y. ; Yamaguchi, K. J. Chem. Phys. 2000, 113, 4035. (b) Onishi, T. ; Takano, Y. ; Kitagawa, Y. ; Kawakami, T.; Yoshioka, Y.; Yamaguchi, K. Polyhedron 2001, 20, 1177-1184. 
The suggestive part of output (including spin populations on $\mathrm{CN}$ suggesting the spin polarization mechanism) is presented in Tables S1 and S2.

Table S1. U-B3LYP data computed for the $a$ and $b$ type of dimers $[\operatorname{Li}(\mu-$ $\mathrm{NC}) \mathrm{FeTp}(\mathrm{CN})(\mu-\mathrm{CN}) \mathrm{Mn}(5-\mathrm{MeO}$ salen $)(\mu-\mathrm{NC}) \mathrm{Li}]^{2+}$

\begin{tabular}{ccccc}
\hline & $\multicolumn{2}{c}{\mathbf{F e}(\mathbf{I I I}) \mathbf{M n}(\mathbf{I I I})}_{\boldsymbol{a}}$ & $\multicolumn{2}{c}{\mathbf{F e}(\mathbf{I I I}) \mathbf{M n}(\mathbf{I I I})}_{b}$ \\
Spin Population & BHS & BLS & BHS & BLS \\
\hline $\mathrm{Fe}$ & 1.0489 & -1.0425 & 1.0480 & -1.0404 \\
$\mathrm{C}$ & -0.0329 & 0.0469 & -0.0224 & 0.0594 \\
$\mathrm{~N}$ & 0.0490 & 0.0114 & 0.0448 & 0.0076 \\
$\mathrm{Mn}$ & 3.8143 & 3.8151 & 3.8101 & 3.8107 \\
& & & & \\
$\left\langle S^{2}\right\rangle$ & 8.7506 & 3.8216 & 8.7506 & 3.8223 \\
& & & & \\
E(a.u.) & -4610.329804 & -4610.329727 & -4610.318262 & -4610.318223 \\
\hline
\end{tabular}

Table S2. U-B3LYP data computed for the effective Mn(III)Mn(III) dimer inside the trimer $\left[\mathrm{CoTp}(\mathrm{CN})_{3}(\mathrm{Mn}(5-\mathrm{MeOsalen}) \mathrm{NCLi})_{2}\right]^{+}$

\begin{tabular}{ccc}
\hline & \multicolumn{2}{c}{ Mn(III)Co(III)Mn(III) } \\
Spin Population & BHS & BLS \\
\hline Mn1 & 3.8212 & 3.8212 \\
Mn2 & 3.8180 & -3.8180 \\
& & \\
$\left\langle S^{2}\right\rangle$ & 20.0009 & 12.3959 \\
& & \\
E(a.u.) & -7080.194846 & -7080.194843 \\
\hline
\end{tabular}

The $\{\mathrm{MnCoMn}\}$ unit was taken to asses the magnitude range of the $J^{\prime}$ next-neighbour interaction, that is in principle comparable with those assignable to the $z J$ interchain effects. The estimation showed a small but sizable amount $J^{\prime}=0.064 \mathrm{~cm}^{-1}$, which, taken qualitatively, is an interesting computation test on the possibility of long range exchange effects. 


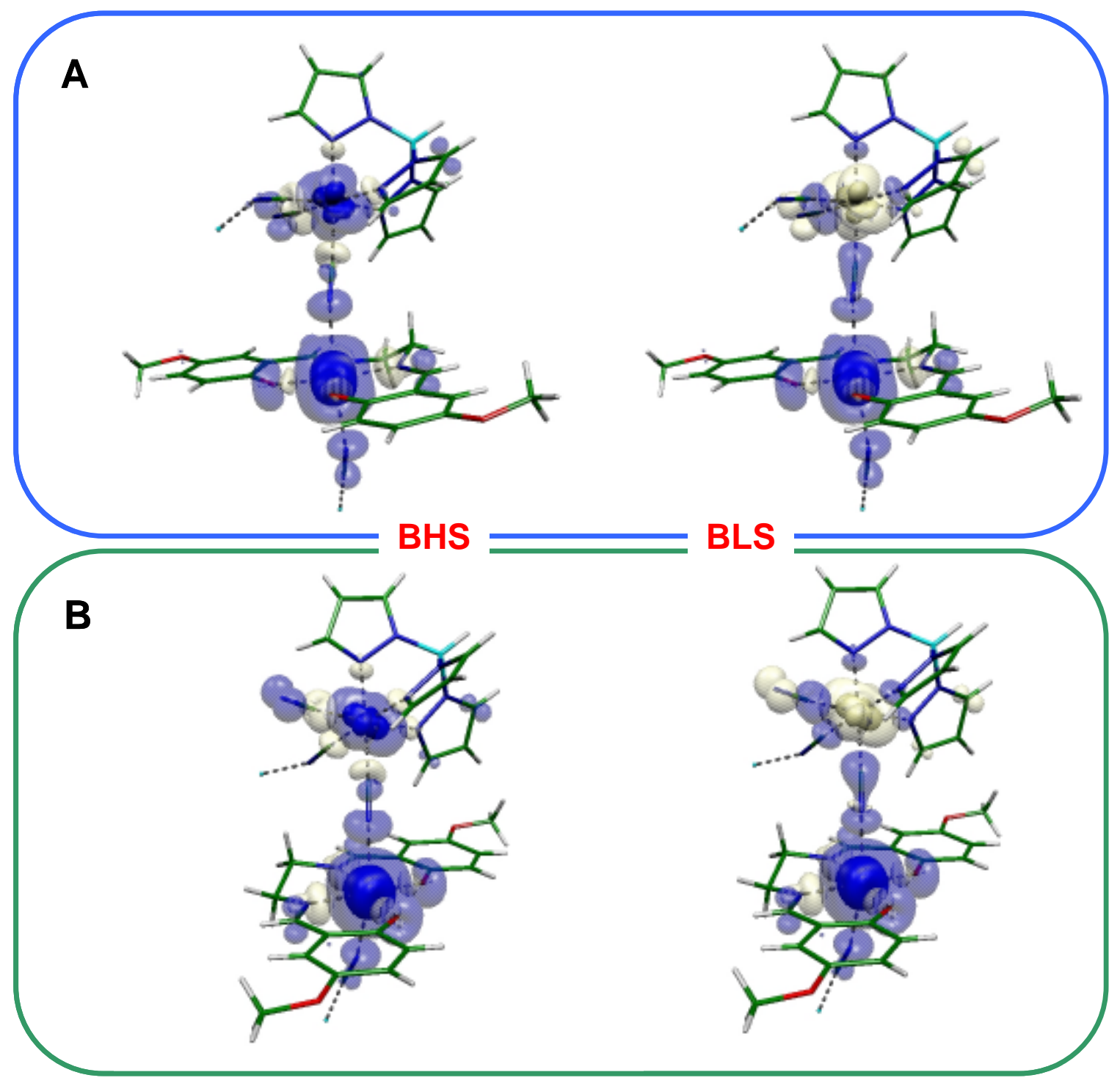

Figure S6. Spin density contours for the BHS and BLS calculations for the $a$ and $b$ dimer sequences ( $\alpha$ in blue and $\beta$ in light yellow). The solid surfaces correspond to the 0.05 $\mathrm{e} / \AA^{3}$ and the semitransparent ones to the $0.002 \mathrm{e} / \AA^{3}$ isovalues. 
The spin natural orbitals processed after the BLS calculations are shown in the figures S.7.and S.8. for the $\{\mathrm{FeMn}\} a$ and $\{\mathrm{FeMn}\} b$ dimer moieties. The $\alpha$ is localized on the $\mathrm{Mn}$ (III) center and the $\beta$ on the $\mathrm{Fe}(\mathrm{III})$ one. These orbitals can be conceived as the magnetic ones.

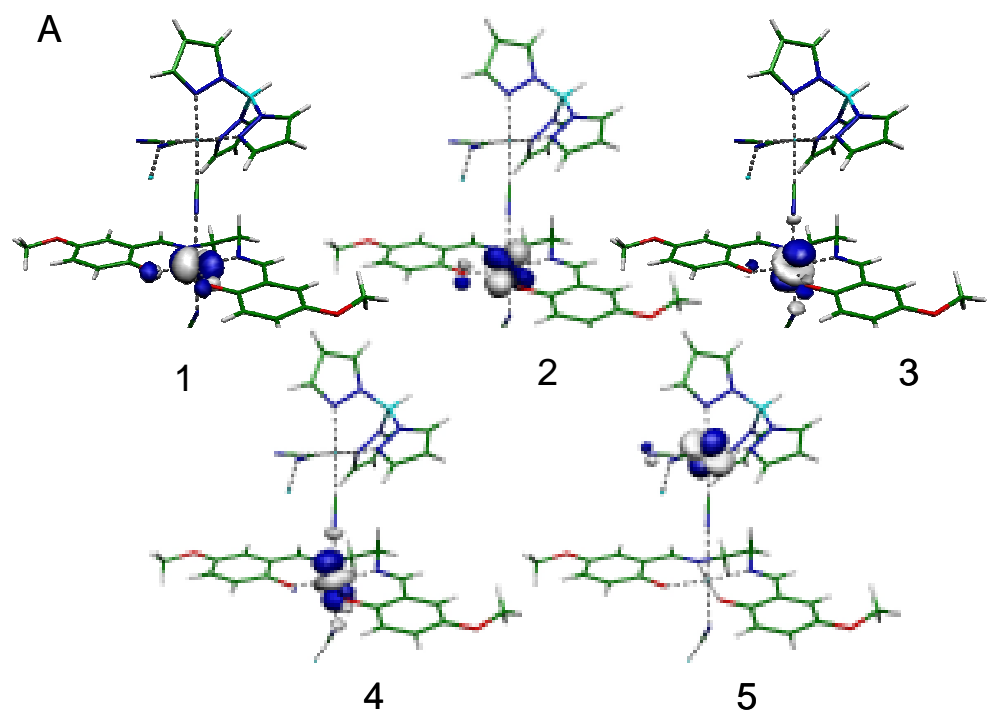

Figure S7. The spin natural orbitals obtained for the BS calculation on the dimer $\{\mathrm{FeMn}\}_{a}$. The orbitals 1-4 have +1 population eigenvalue $(\alpha)$ and the orbital 5 shows -1 population eigenvalue $(\beta)$.
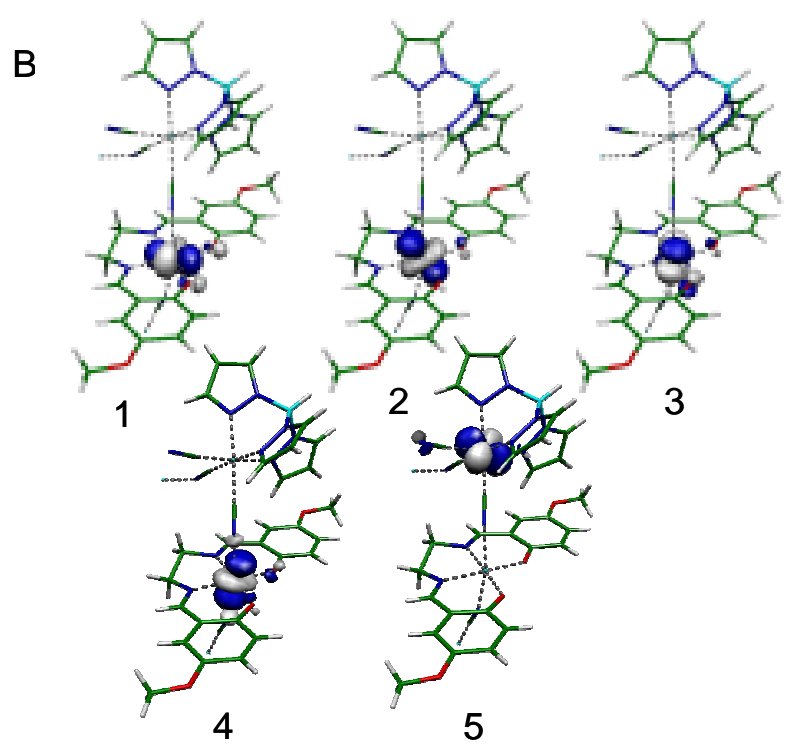

Figure S8. The spin natural orbitals obtained for the BS calculation on the dimer $\{\mathrm{FeMn}\}_{b}$. The orbitals 1-4 have +1 population eigenvalue $(\alpha)$ and the orbital $\mathbf{5}$ shows -1 population eigenvalue $(\beta)$. 
If formally set the $x y$ axes closely to the $\mathrm{N}-\mathrm{Mn}-\mathrm{O}$ mean directions, the orbitals look like a certain $d$-AOs mutual mixing taken over the set $\left\{d_{\mathrm{xz}}, d_{\mathrm{yz}}, d_{\mathrm{xy}}, d_{\mathrm{z} 2}\right\}$. Thus, orbital placed at 1 labels in the above figure sections are approximately the $d_{\mathrm{xy}} \equiv d_{\pi \|}$ equatorial AO, those in $\mathbf{2}$ and 3 approximately $\left(d_{\mathrm{xz}} \pm \cdot d_{\mathrm{yz}}\right) / \sqrt{2} \equiv \mathrm{d}_{\pi \perp}$ axial $\pi$ AOs and the $\mathbf{4}$ close to a $d_{\mathrm{z} 2}=d_{\sigma}$ axial $\sigma \mathrm{AO}$.

It is interesting to note, in the orbital shapes, a certain mixing between axial $d_{\sigma}$ and $d_{\pi \perp}$ pure components due to the slight $\sigma-\pi$ mixing induced by the nonlinearity of $\mathrm{CN}$ bridges. The magnetic orbital on the Fe(III) part belongs to the $d_{\pi}$ type. The exchange channel between orbitals $\mathbf{4}$ and $\mathbf{5}$ is qualitatively creditable as the higher in magnitude, because of extension of $\mathbf{4}$ toward the next center, along the bridge, and of ferro type, because of $d_{\sigma}(\mathrm{Mn})-d_{\pi}(\mathrm{Fe})$ orthogonality relationship. The other $d_{\pi}(\mathrm{Mn})-d_{\pi}(\mathrm{Fe})$ interactions (orbitals 1-3 against 5) are antiferro channels, but conceivable with lower magnitude because of intrinsic small $\pi$ - $\pi$ overlap.

The spin density presented in Fig.4 and Fig. S6 approximately corresponds to the summation of densities of orbitals 1-4 for the Mn(III) moiety and to the contour of orbital 5 on the Fe(III) complex side. 